\title{
A Reduced Model of a Thermo-Elastic Nonlinear Circular Plate
}

\author{
Anna Warminska ${ }^{1, \star}$, Emil Manoach ${ }^{2, \star \star}$, and Jerzy Warminski ${ }^{3, \star \star \star}$ \\ ${ }^{1}$ Department of Thermodynamics, Fluid Mechanics and Aviation Propulsion Systems, \\ Lublin University of Technology, Lublin, Poland \\ ${ }^{2}$ Institute of Mechanics, Bulgarian Academy of Sciences, Sofia, Bulgaria \\ ${ }^{3}$ Department of Applied Mechanics, Lublin University of Technology, Lublin, Poland
}

\begin{abstract}
Nonlinear vibrations of a circular plate subjected to mechanical and thermal loadings are presented in the paper. A model of the plate is based on the extended Mindlin theory, taking into account nonlinear geometrical terms and acting heat uniformly distributed along the plate span. The dynamics of a coupled thermo-mechanical problem is reduced from a set of partial differential equations to ordinary differential equations. Considering oscillations around the first natural frequency just one mode reduction is proposed. The analysis shows that elevated temperature shifts the resonance curve and new post-buckling oscillations arise. Depending on initial conditions for the post-buckling state various scenarios of bifurcations take place and transient irregular oscillations may occur. The proposed one degree of freedom model shows a good agreement with response of the model based on three or five-modes reduction.
\end{abstract}

\section{Introduction}

The ambient temperature which may vary in a large range may essentially influence dynamics of mechanical, aviation or aerospace structures, starting from low negative, up to high positive temperature values. The variation of temperature may be crucial while performing experimental tests. In some cases, close to critical points, even small temperature variation may give unexpected change in the system response. The fundamentals of thermoelastic problems with aerospace engineering applications have been published in [1]. In publications [2] and [3] thermo-elastic vibrations of beam element have been studied in context of elevated abient temperature or heat flux acting directly on the beam. It has been shown that heat flux may change the transient as well as the steady state system's response. The coupled fields problem, represented by a set of partial differential equations (PDE) has been solved numerically by proposed by authors iterative procedure based on pseudo-normal modes approach. Reduced models of a nonlinear Timoshenko beam model have been presented in [4], [5]. The model has been obtained by just one mode reduction for the case of the elevated and uniformly distributed temperature. The influence of elevated temperature on the resonance curves and bifurcation points has been presented. The resonance curves have been shifted into lower frequencies if the temperature has been increased. Furthermore, above the certain threshold, the increased temperature has led to bucking phenomenon and small oscillations around a new post-

\footnotetext{
^e-mail: a.warminska@pollub.pl

$\star \star$ e-mail: e.manoach@imbm.bas.bg

$\star \star \star$ e-mail: j.warminski@pollub.pl
}

buckled state or large chaotic oscillations around the neutral position. The thermo-elastic Timoshenko beam model has been extended for a nonuniform distribution of the temperature [6]. The reduced model allowed bifurcation analysis for nonuniform heat distribution.

Thermally induced oscillations of 2D structures e.g rectangular nonlinear plate models based on higher order shear deformation theory have been analysed in [7], [8]. The post-buckling phenomenon for isotropic or composite plates has been presented for different coefficient of thermal expansion and geometric imperfections. The theoretical results have been validated by experimental tests. Influence of temperature on moderately thick functionally graded plates is presented in [9]. Both material properies and the temperature have been assumed as varied nonlinearly through the plate thickness. Complex dynamics including periodic, quasi-periodic and chaotic motions have been found. The bifurcation analysis has been based on a large number of degrees of freedom which caused numerical complications, comparing with reduced low dimensional models. In recent publications [10], [11] a 2D continuum model of the nonlinear dynamic problem for a von Karman shear indeformable symmetric cross-ply laminated plate in a thermomechanical environment has been presented. The main features of nonlinear effects coupled with thermal field have been systematically analysed. A comparison of response by partially coupled and uncoupled mechanical reduced models has been demonstrated. The reduced model has consisted of one mechanical and two thermal ordinary differential equations. The reduced model preserved the main features of the full represented by PDEs model. The systematic numerical investigations 
of local and global dynamics of the plate for varying thermal and mechanical loadings have been presented.

The analysis performed for circular plate at elevated temperature and selected variants of external excitation has been presented in $[6,12]$. The exact thermomechanical model of the circular plate described by PDEs presented has been reduced to one degree of freedom system. The preliminary results demonstrated thermally induced buckling phenomenon and then the post-buckling bifurcation scenario. In this paper we check correctness of the model proposed in paper [12] comparing one mode reduction with numerical simulations based on several pseudo-modes reduction.

\section{A model of circular plate}

A nonlinear model of the plate is based on extended Mindlin plate theory. The model proposed in this paper takes into account nonlinear displacement field, thermal and mechanical loadings. The circular plate taken under the study is presented in Fig. 1.

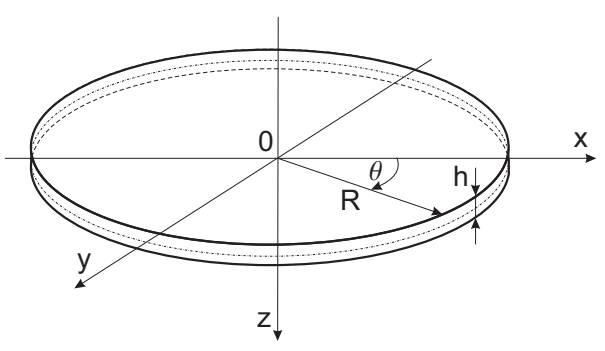

Figure 1. Circular plate model with indicated coordinates and dimensions.

The mathematical model of a fully coupled thermomechanical model is represented by a set of partial differential equations. The axis-symmetric vibrations of a nonlinear thermo-elastic plate has the form [12]

$$
\begin{aligned}
& A h\left[\frac{\partial^{2} u}{\partial r^{2}}+\frac{\partial w}{\partial r} \frac{\partial^{2} w}{\partial r^{2}}+\frac{1}{r} \frac{\partial u}{\partial r}-\frac{u}{r^{2}}+\frac{1}{2 r}(1-v)\left(\frac{\partial w}{\partial r}\right)^{2}\right] \\
& =A(1+v) \alpha_{T} \frac{\partial \gamma_{T}}{\partial r} \\
& D\left[\frac{\partial^{2} \psi}{\partial r^{2}}+\frac{1}{r} \frac{\partial \psi}{\partial r}-\frac{\psi}{r^{2}}\right]-k^{2} G h\left(\frac{\partial w}{\partial r}+\psi\right)-c_{2} \frac{\partial \psi}{\partial t}-\frac{\rho h^{3}}{12} \frac{\partial^{2} \psi}{\partial t^{2}} \\
& =A(1+v) \alpha_{T} \frac{\partial \kappa^{T}}{\partial r}
\end{aligned}
$$$$
k^{2} G h\left(\frac{\partial^{2} w}{\partial r^{2}}+\frac{1}{r} \frac{\partial w}{\partial r}+\frac{\partial \psi}{\partial r}+\frac{\psi}{r}\right)
$$$$
+A h\left[\frac{\partial u}{\partial r}+\frac{1}{2}\left(\frac{\partial w}{\partial r}\right)^{2}+\frac{v}{r} u-\frac{1+v}{h} \alpha_{T} \gamma_{T}\right]\left(\frac{\partial^{2} w}{\partial r^{2}}+\frac{1}{r} \frac{\partial w}{\partial r}\right)
$$$$
+A h\left(\frac{\partial^{2} u}{\partial r^{2}}+\frac{v}{r} \frac{\partial u}{\partial r}-\frac{v}{r^{2}} u+\frac{\partial w}{\partial r} \frac{\partial^{2} w}{\partial r^{2}}-\frac{1+v}{h} \alpha_{T} \frac{\partial \gamma_{T}}{\partial r}\right) \frac{\partial w}{\partial r}
$$$$
+c_{1} \frac{\partial w}{\partial t}-\rho h \frac{\partial^{2} w}{\partial t^{2}}=-p(r, t)
$$

where $u(r, t)$ is the in-plane displacement, $w(r, t)$ is the transverse displacement, $\psi(r, t)$ is the crosssection rotation angle and $h$ and $R$ are the plate thickness and radius. Material parameters are defined by the Young and shear modulus $E, G$, material density $\rho$, the coefficient of thermal expansion $\alpha_{T}$. The rest of coefficients and functions is defined as: $A=\frac{E}{1-v^{2}}, D=\frac{E h^{3}}{12\left(1-v^{2}\right)}, \gamma^{T}(r)=\int_{-h / 2}^{h / 2} \Delta T(r, z) d z$, $\kappa^{T}(r)=\int_{-h / 2}^{h / 2} \Delta T(r, z) z d z, \Delta T=T-T_{0}$, where $T$ and $T_{0}$ are current and reference temperatures and $p(r, t)$ is external loading function. One can note that in Eq. (1) the inertia term $\frac{\partial^{2} u}{\partial t^{2}}$ is neglected. The above equations can be solved numerically by applying pseudo-modes method or reduced to ordinary differential equations taking a properly selected number of modes.

\section{Circular plate reduced model}

The full model of the plate represented by partial differential equations is reduced to the ordinary differential equations by the Galerkin method based on the modes projection. We assume that the temperature is uniformly distributed along the plate span and thickness and is equal to ambient temperature. In such a case Eq. (1) is solved analytically and coordinate $u$ is related to $w$. Assuming solution in the series

$$
w(r, t)=\sum_{n=1}^{N} q_{n}(t) W_{n}(r), \quad \psi(r, t)=\sum_{n=1}^{N} q_{n}(t) \Psi_{n}(r)
$$

where $W_{n}(r), \Psi_{n}(r)$ are assume vibration modes and $q_{n}(t)$ generalised coordinates, the PDEs are transformed to ordinary differential equations (ODE) by the Galerkin method. The modes should satisfy geometrical boundary conditions. We take into consideration a case of the clamped plate. The mode shapes can be approximated by the Bessel functions which satisfy boundary conditions. The first there modes against radius normalised to one are presented in Fig. 2.

In this paper we take one mode reduction of the clamped plate, considering excitation distributed according to the first mode shape. Substituting solution Eq. (2) into PDEs, multiplying by the the selected mode function and integrating through the plate radius, we get a set of differential equations of motion. For a first mode reduction we obtain one degree of freedom nonlinear model

$\ddot{q}_{1}+2 \xi_{1} \omega_{1} \dot{q}_{1}+\omega_{1}^{2} q_{1}+F_{N L 1} q_{1}^{3}+F_{N T 1} \Delta T q_{1}=F_{N P 1} \sin \omega t$.

The above equation is written in the form where time is given in seconds but displacements are represented in dimensionless form, $\bar{u}=u / R, \bar{w}=w / R$. The 'dot' denotes time derivative and $q_{1}$ dimensionless generalised coordinate, $\omega_{1}$ means the first natural frequency, $F_{N L 1}$ is nonlinear geometrical term coming from the original model, $F_{N T 1}$ is a coefficient related to temperature variation, $F_{N P 1}$ means amplitude of mechanical loading. Modal damping is defined by coefficient $\xi_{1}$. 

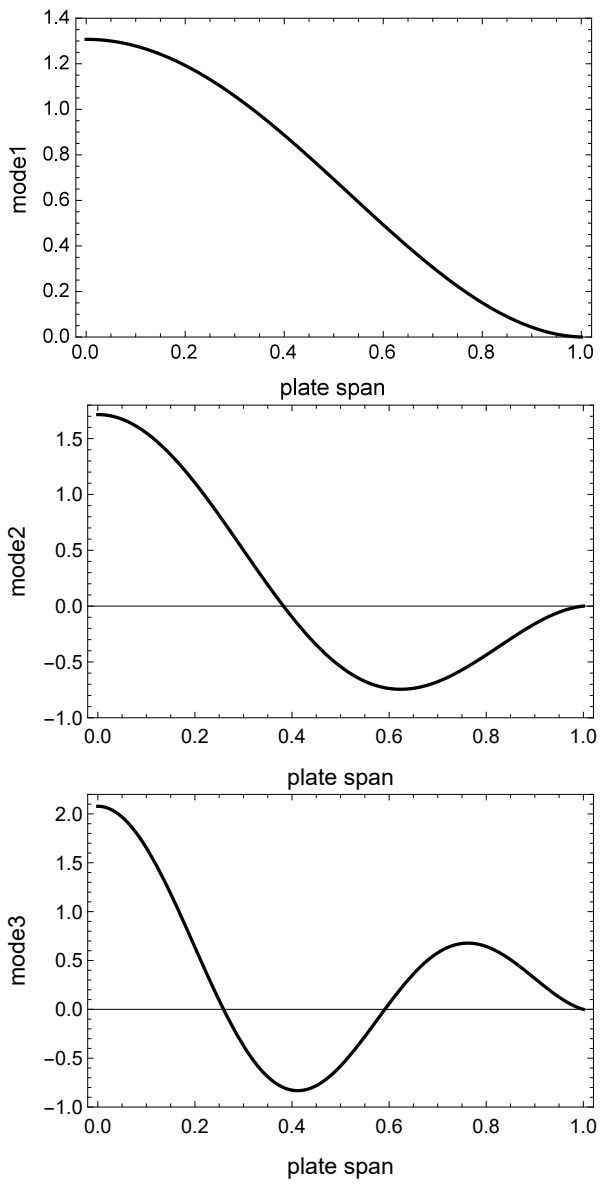

Figure 2. The first three vibration modes of the clamped circular plate described by the modified Bessel functions.

\section{Numerical analysis of the reduced model}

Example of numerical studies is performed for the clamped circular plate with diameter: $R=0.1 \mathrm{~m}$, and thickness $h=0.0025 \mathrm{~m}$. The plate has homogenous properties and following material data: $E=0.7 \times 10^{11} \mathrm{~Pa}$, $v=0.34, \rho=2778 \mathrm{~kg} / \mathrm{m}^{3}$. The reduced model coefficients computed on the basis of the physical parameters after the modal reduction procedure take values:

$$
\begin{aligned}
& \omega_{1}=3928.1, \xi_{1}=0.01, F_{N L 1}=0.29497 \times 10^{11}, \\
& F_{N T 1}=-0.358635 \times 10^{7}, F_{N P 1}=0.30849 \times 10^{4}
\end{aligned}
$$

On the basis of the reduced analytical model Eq. (3) we computed a resonance curve for $\Delta T=0$. It means that the temperature does not change with respect to the reference one. The plate is excited by harmonic loading with fixed amplitude and excitation frequency varied around the first natural frequency. In Fig. (3) the resonance curve demonstrates nonlinear behaviour with evident stiffening phenomenon. The solid and dashed lines represent stable and unstable solutions, respectively.

The influence of the elevated temperature on the plates response we can check by fixing mechanical excitation and then varying the temperature with respect to the reference one. Therefore we select from Fig. (3) frequency

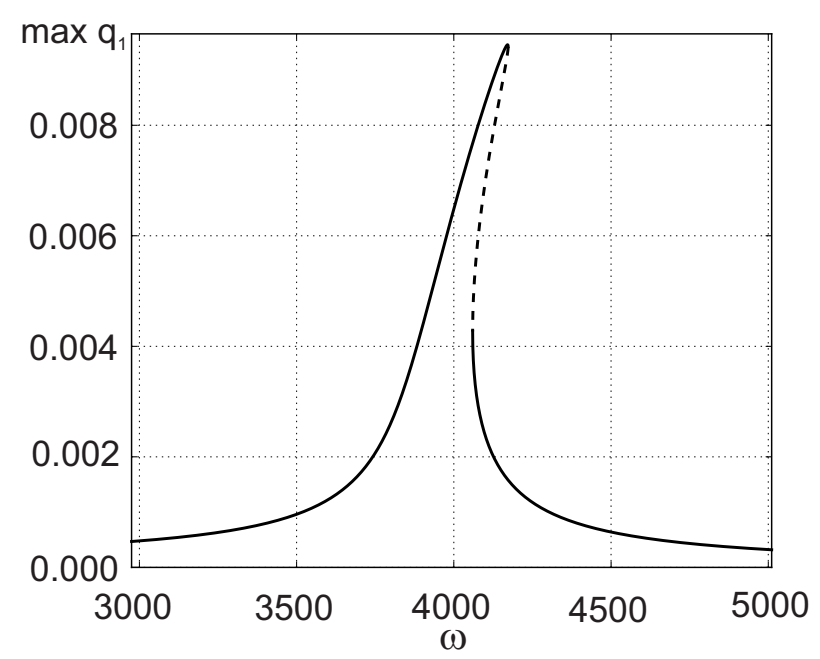

Figure 3. Resonance curve of the reduced model, $\Delta T=0$, $F_{N P 1}=0.30849 \times 10^{4}$.

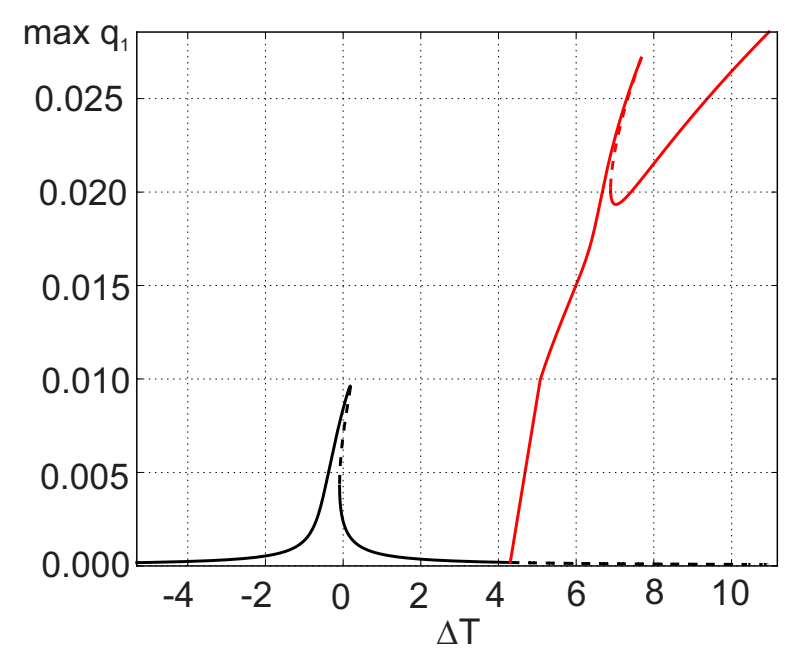

Figure 4. Bifurcation diagram of coordinate $q_{1}$ against temperature variation $\Delta T, F_{N P 1}=0.30849 \times 10^{4}, \omega=4100$.

$\omega=4100$, which corresponds to a multi-solution case with stable, large or small, amplitudes and unstable solution between them. The bifurcation diagram for the fixed mechanical loading and varying temperature is presented in Fig. (4). In fact for $\Delta T=0$ we observe three different solutions, corresponding to diagram Fig. (3). However, if temperature increases above the bifurcation point $D T=4.3$ the buckling of the plate occurs, and the plate oscillates around post bucking state. The post-buckling oscillations are represented by the red curve in Fig. (4). The corresponding time history for post-buckling oscillations for $\Delta T=6$, is presented in Fig. 5. The buckling phenomenon is observed by offset of the oscillation centre, and depending on initial conditions, it can buckle in a positive or negative direction.

In order to get knowledge how precise is just one mode reduction we compare the results with three and five modes models obtained from numerical simulation of exact PDEs and using the selected number of modes. 


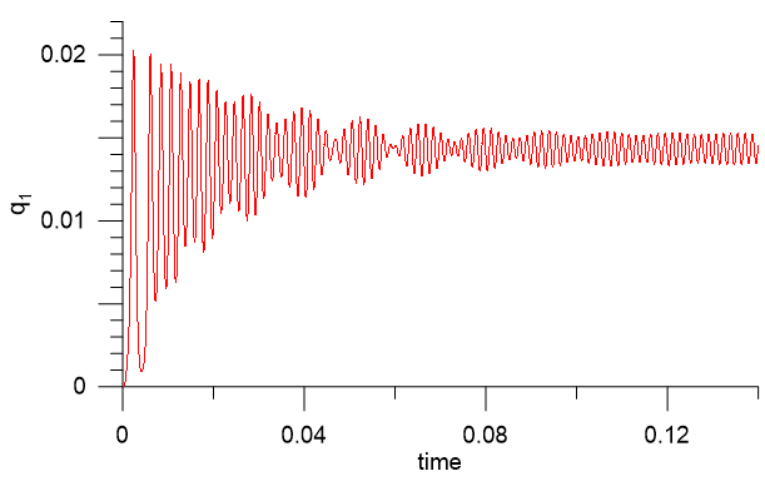

Figure 5. Time history for temperature $\Delta T=6$, the solution obtained for 1 mode reduction; $\omega=4100$.

The main assumption, however is that the plate is excited around the first natural frequency.

(a)

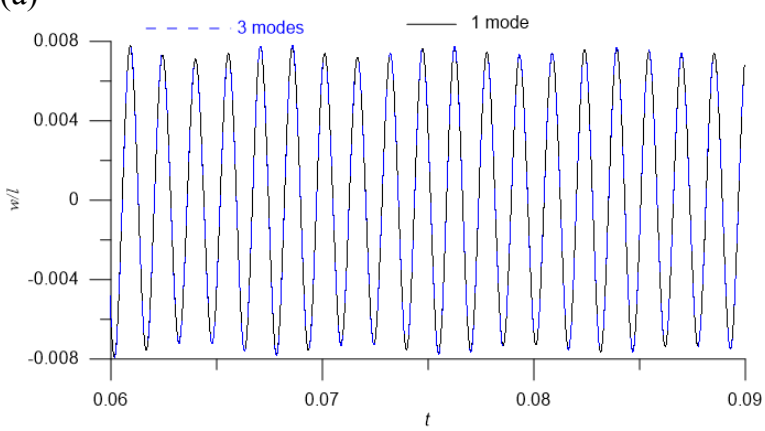

(b)

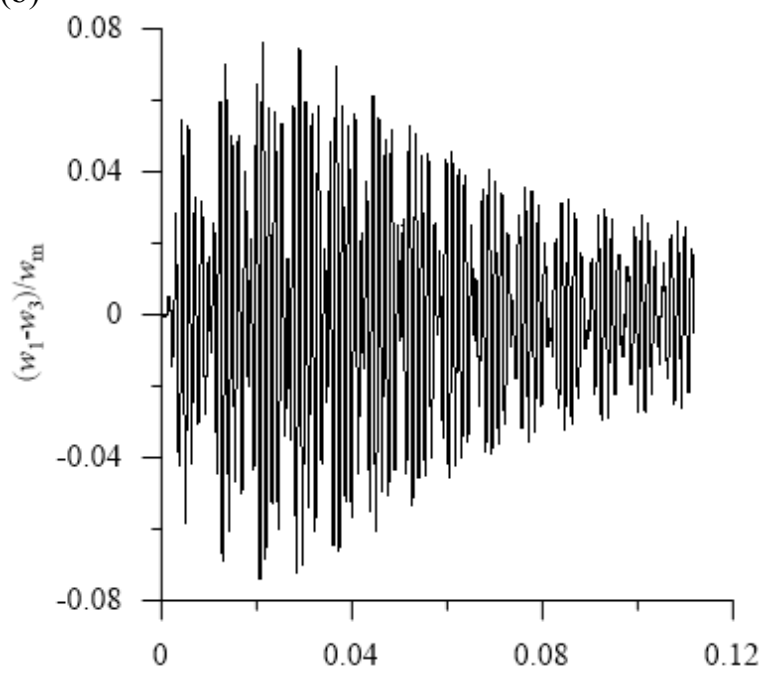

Figure 6. Time histories for temperature $\Delta T=0$, (a) solution obtained for 1 mode (black) and 3 modes (blue) reduction; (b) time history of relative difference; $\omega=4100, w_{m}=0.0025$.

The example of the comparison of the model based on three and five modes with the one mode reduction is presented in Fig. 6 (a) and Fig. 7 (a) for $\omega=4100$ and $\Delta T=0$ and large oscillations corresponding to upper branch in Fig. 3. The relative difference between free $\left(w_{3}\right)$ or five $\left(w_{5}\right)$ modes reductions and one mode model $\left(w_{1}\right)$, ex- (a)

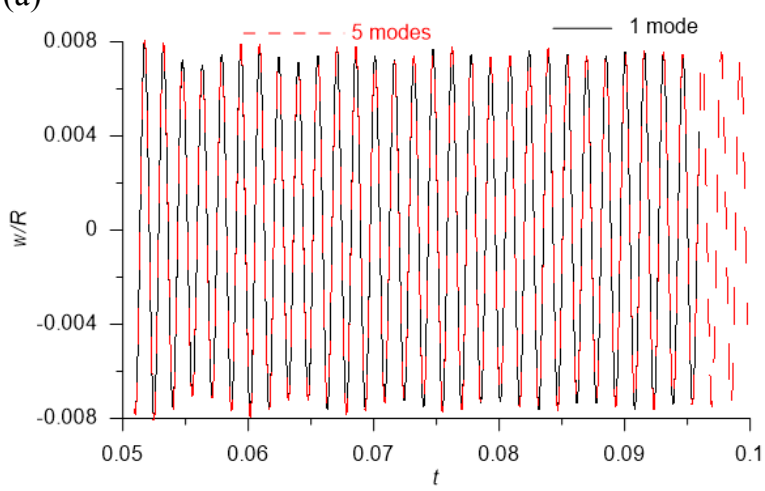

(b)

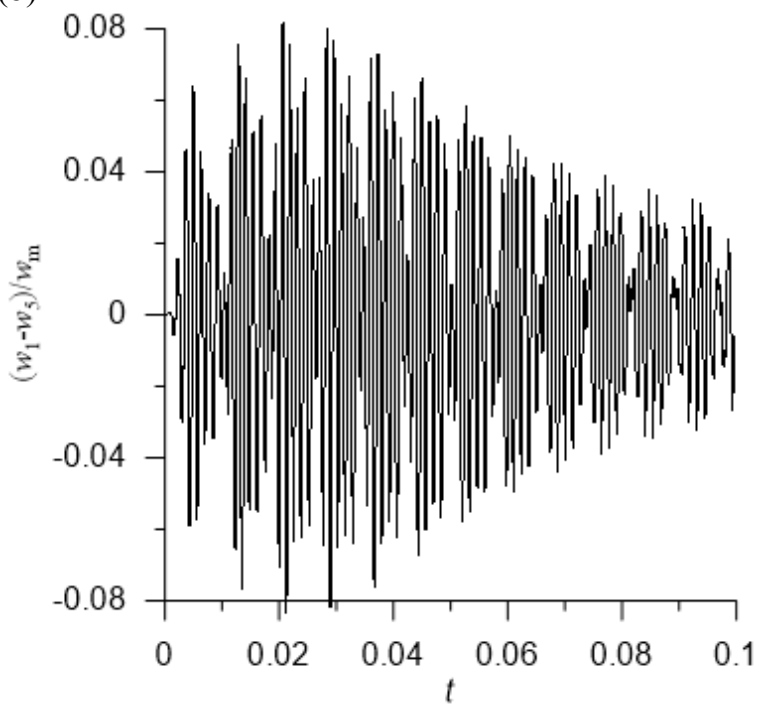

Figure 7. Time histories for temperature $\Delta T=0$, (a) solution obtained for 1 mode (black) and 5 modes (red) reduction; (b) time history of relative difference; $\omega=4100, w_{m}=0.0025$.

pressed versus mean value $\left(w_{m}\right)$, is presented in Fig. 6 (b) and Fig. 7 (b), respectively. We note that for transient motion the difference is less than $8 \%$ and less then $4 \%$ for a steady state. In other cases for smaller amplitudes the difference is even smaller. Thus, the one degree of freedom model can be successfully implemented for detailed bifurcation analysis and only selected solutions verified by simulation of the exact model, which of course is more demanding and time consuming process.

The elevated temperature may lead to very complex response of the system. The bifurcation diagram in Fig. 8 demonstrates possible multi steady state solution (lines) or irregular oscillations (dark region) if temperature elevates up to $\Delta T=5$. The detailed analysis of the Poincare section confirmed that the dark region represents transient irregular oscillations which after long time motion become periodic.

\section{Conclusions}

The extended model of Mindlin plate with geometrical nonlinearities which occur due to large deformations is presented in the paper. The model takes into account 


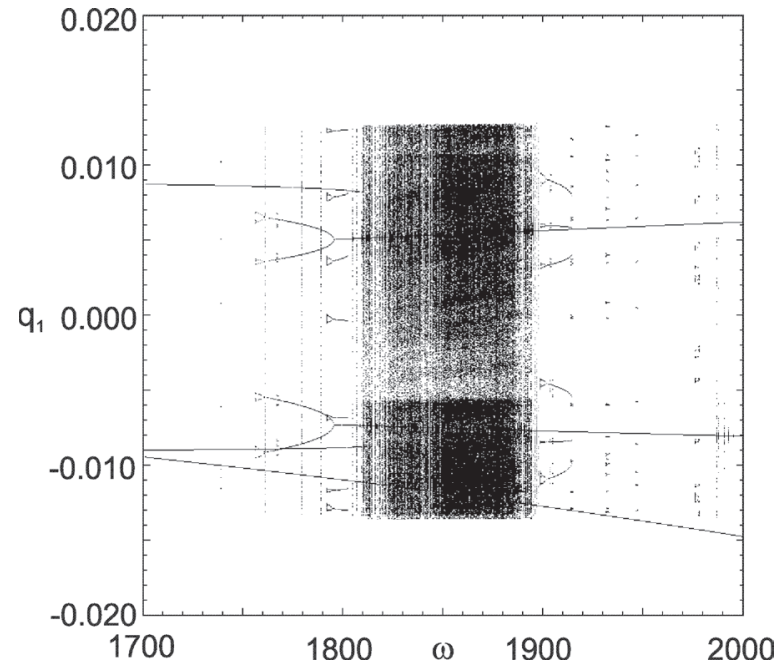

Figure 8. Bifurcation diagram of coordinate $q_{1}$ versus excitation frequency, $\Delta T=5, F_{N P 1}=0.30849 \times 10^{4}$.

temperature which is assumed to be uniformly distributed along the plate spam. The exact model represented by PDEs is reduced to one degree of freedom system taking into account the nonlinear geometrical terms and temperature contribution. It is shown that one mode reduction can be successfully applied around the first resonance region, and the relative difference between one-mode and three or five-modes reduction is small. Thus, the proposed one degree of freedom nonlinear model can be used for effective bifurcation analysis.

The simulations based on the first mode reduction exhibit the stiffening effect of the resonance curve. The elevated temperature leads to bifurcation and buckling phenomenon and then post-buckling oscillations around a new steady state. The post-buckling oscillations are characterised by the positive or negative offset. The bifurcation analysis for elevated temperature shows multi-solutions, period doubling bifurcations and the transient irregular motion (transient chaos)which, after long time, goes to the periodic solution.

\section{Acknowledgements}

The first author would like to acknowledge the support received from the Polish NSF Grant S-38/M/2017, the second author the partial support from Bulgarian NSF Grant DN 02/7 2016 and H2020 Marie Skłodowska-Curie ITN under grant agreement No 643095, and the third author the support from Polish NSF grant S-3/M/2017.

\section{References}

[1] E.A. Thorton, Thermal structures for aerospace applications (AIAA Education Series, 1996) 1-479.

[2] E. Manoach, P. Ribeiro, Int. J. Mech. Sci., 46, (2004) 1589-1606.

[3] P. Ribeiro, E. Manoach, J. Sound and Vibration, 285, (2005) 1093-1107.

[4] A. Warminska, E. Manoach, J. Warminski, Meccanica, 49, (2014) 1775-1793.

[5] A. Warminska, E. Manoach, J. Warminski, S. Samborski, Continuum Mechanics and Thermodynamics, (2014), DOI 10.1007/s00161-014-0381-6.

[6] A. Warminska, E. Manoach, J. Warminski, Proceedings of ICoEV 2015 Int. Conf. on Engineering Vibration, Ljubljana, Slovenia, (2015) 1057-1066.

[7] M. Amabili, S. Carra, J. Sound and Vibration, 321, (2009) 936-954.

[8] M. Amabili, M. Reza, S. Tajahmadi, J Mechanical Engineering Science (2012) 226 2393-2401.

[9] F. Alijani, F. Bakhtiari-Nejad, M. Amabili, Nonlinear Dynamics (2011) 66 251-270.

[10] E. Saetta, G. Rega, Composite Structures, 174, (2017) 420-441

[11] E. Saetta, G. Rega, Nonlinear Dynamics, 174, (2017) DOI 10.1007/s11071-017-3648-1

[12] A. Warminska, E. Manoach, J. Warminski, Book Series: MATEC Web of Conferences, 83, (2016) UNSP 05013 